\title{
How Flat is Flat? Measuring Payoff Functions and the Implications for Site-Specific Crop Management
}

\author{
Dave Pannell \\ School of Agriculture and Environment \\ University of Western Australia \\ Perth WA 6009, Australia \\ David.Pannell@uwa.edu.au \\ ORCID: 0000-0001-5420-9908
}

Markus Gandorfer

Institute for Agricultural Engineering and Animal Husbandry

Bavarian State Research Center for Agriculture

Vöttinger Str. 36, D-85354 Freising, Germany

markus.gandorfer@Ifl.bayern.de

ORCID: 0000-0002-0624-153X

Alfons Weersink (corresponding author)

Dept of Food, Agricultural and Resource Economics

University of Guelph,

Guelph, ON, Canada N1G 2W1

aweersin@uoguelph.ca

1-519-824-4120 ext 52766

ORCID:0000-0001-5081-3593

\begin{abstract}
Within the neighbourhood of any economically "optimal" management system, there is a set of alternative management systems that are only slightly less attractive than the optimum. Often this set is large; in other words, the payoff function is flat within the vicinity of the optimum. This has major implications for the economics of variable-rate site-specific crop management. The flatter the payoff function, the lower the benefits of precision in the adjustment of input rates spatially within a crop field. This paper is about how we can best measure the flatness of payoff functions, in order to assist with judgements about the likely benefits of site-specific crop management. We show that two existing metrics - the relative range of an input for which the payoff is at least $95 \%$ as large as the maximum payoff (IR95) and the relative curvature (RC) of the payoff function - are flawed. We suggest an alternative metric: the standard deviation of the slopes of site-specific payoff-functions at the optimal uniform input rate (SDS). The SDS is highly correlated with the benefits from variablerate precision management.
\end{abstract}

\section{Highlights}

- The flatter the payoff function, the lower the benefits of precision agriculture

- Existing metrics of flatness of the payoff function are flawed.

- A new metric is correlated with the benefits for variable rate nitrogen application.

Keywords: Payoff function, curvature, flatness measures, optimal nitrogen rates 


\section{Introduction}

Payoff functions for agricultural inputs are generally flat (Pannell 2006). A payoff function is the relationship between an input rate and profit per unit area or another measure of economic benefit. A flat payoff function means that, at input rates somewhat above or below the optimum, the payoff to farmers is only slightly less than the payoff at the optimal input rate. Pannell (2006) showed that this has a range of implications for the economics of farm management, including for precision technologies that allow site-specific crop management. The flatter the curve, and the wider the input range over which it is flat, the lower the benefit from adjusting input rates spatially in response to local conditions. Precision agriculture allows input application to be tailored to match the differing needs across a field, but the value of the enhanced accuracy may not be worth the cost (Weersink et al. 2018). Emphasising this point, before precision technologies had been conceived, Anderson (1975, p.195) commented that "In pursuing ... optimal levels of decision variables, precision is pretence and great accuracy is absurdity".

Crop fields with high spatial heterogeneity of yield are commonly thought to have high potential benefits from site-specific crop management. However, high yield heterogeneity does not necessarily result in high economic benefits of variable-rate site-specific crop management (e.g., Lawes and Roberstson 2011; Bachmaier and Gandorfer 2009). If the payoff functions for management zones within a field are sufficiently flat, the benefits of adjusting rates to best suit the yield potential for each part of the field can be very low.

Although Pannell (2006) suggests flat payoff functions from the application of inputs in agriculture are the norm, the flatness of the payoff curve varies to some degree, from case to case. This suggests a strategy of measuring the degree of flatness of payoff curves, in order to identify situations where the benefits of site-specific crop management are more likely to be high. More accurate information about flatness may contribute to decisions by farmers about their investment in precision technologies, may assist precision-technology researchers to target their efforts to the 
1 most promising contexts (e.g. regions, crops or soil types), or may assist technology sellers to target

2 their sales activities to contexts where they are most likely to succeed. Two flatness metrics have been used in the literature. The first, defined by Pannell (2006), is based on the range of an input within which the payoff is at least $95 \%$ as large as the maximum payoff. Rogers et al. (2016) proposed a second metric, which they termed relative curvature (RC). Rogers et al. (2016) argued that RC is superior to the input range indicator proposed by Pannell (2006), but they did not compare the economic performance of each when used as a guide to decision making. Without such a comparison, their usefulness in assessing the profitability of precision agriculture is unknown. The purpose of this paper is to evaluate various metrics for measuring the flatness of the payoff function in the hope of identifying a simple option that reflects the economic benefits of sitespecific crop management reasonably accurately. The paper begins by describing the two current measures of flatness in the site-specific management literature and proposes a third metric that recognises the different payoff functions in different areas of a field. The next section develops an economic model to estimate the benefits of variable-rate site-specific management followed by a description of its empirical application. Following this, the benefits of variable-rate site-specific management are calculated for various scenarios and compared with the three flatness metrics. These measures of flatness for the payoff functions can be used across a variety of situations to determine the value of the additional information from greater precision from input application in agriculture.

Materials and Methods

\section{Measures of Flatness}

Pannell (2006) used a simple but ad hoc indicator of flatness: the range of input level $(x)$ for which the payoff $\pi(x)$ is at least $95 \%$ as large as the maximum payoff $\pi_{\max }=\pi\left(x^{*}\right)$, where $x^{*}$ is the input 
1 level that maximises the payoff function. Although this was not proposed as an indicator for decision

2 making, it could potentially be used as such. Normalised to $x^{*}$, this indicator is:

where IR95 stands for the Input Range $95, \underline{x}_{u}$ is the upper limit of the range of input levels that result in a payoff of at least $0.95 \times \pi_{\max }$, and $x_{d}$ is the downside limit of that range. The variables used to calculate IR95 are shown in Figure 1.

Rogers et al. (2016) proposed a second measure of the flatness of a payoff curve, which they termed relative curvature $(R C)$.

where is an arbitrary input level that sets the upper range for measuring $R C$. The calculation of $R C$ is illustrated in Figure 2. It is equal to the shaded area divided by the area of the rectangle . Rogers et al. (2016) found that relative curvature varies substantially between cases, and suggests that it be used to identify those fields where site-specific crop management should be applied. In addition to IR95 and $R C$, we evaluate a third measure: the standard deviation of the slopes of site-specific payoff-functions at the optimal uniform input rate, $x^{*}$ (SDS for short). This measure recognises that there are different payoff functions in different areas of a field. We assume that the field can be broken into $n$ areas, each of which is uniform within that area. Each area has a different payoff function for the input. The overall payoff function for a uniform input rate is the weighted combination of the payoff functions for the $n$ parts of the field. From that overall payoff function, we determine the optimal uniform input rate for the field, $x^{*}$. Then, for each part of the field, we determine the slope of the payoff function at $x^{*}$, which, for the ith part of the field, we represent as $\pi_{i}^{\prime}\left(x^{*}\right)$. Then we calculated the standard deviation of those slopes across the field, SDS:

where is the mean slope of the payoff function across the $\mathrm{N}$ different areas of the field. The calculation of standard deviation is weighted by the area for which each payoff function applies. 
1 Figure 3 shows a simple illustrative example, where $n=3$. The tangents at $x^{*}$ for each of the three payoff functions are shown. SDS is the standard deviation of the slopes of these tangents. The reason for testing this metric is that the slope of the payoff function at $\mathrm{x}^{*}$ indicates the potential gain in payoff from adjusting the input rate away from the optimal uniform level, and that it reflects the heterogeneity of the field, which underpins the gains from site-specific management.

\section{Economic Benefits of Site-Specific Crop Management}

In order to evaluate the suitability of the three flatness metrics for indicating the economic benefits of site-specific crop management, an economic model of site-specific crop management is needed.

We develop the model assuming nitrogen is the input for the simple illustrative case where $n=3$ with $\mathrm{l}=\mathrm{low}, \mathrm{m}=$ medium and $\mathrm{h}=$ high, but it is generalizable to any $\mathrm{n}$.

$$
\pi_{n}=P_{y} F_{n}(N)-P_{N} N_{n} \quad(n=l, m, h)
$$

where $\pi_{n}$ is the payoff to area $n$ per unit of land area, $P_{y}$ is the price of the crop per tonne, $F_{n}(N)$ is the production function relating the nitrogen application rate $\left(N_{n}\right)$ to the quantity of crop produced per unit of land area, and $P_{N}$ is the price of nitrogen. The first-order condition for maximizing the payoff function is where the marginal benefit of applying an extra unit of nitrogen (output price times the extra unit of output stemming from the extra amount of fertilizer) is equal to the marginal cost of nitrogen or its price;

$$
P_{y}\left(F_{n}(N) / N\right)=P_{N} \quad(n=1, m, h)
$$

The optimal rate is found by solving the above first-order condition explicitly for $\mathrm{N}$. For example, if the following quadratic production function was assumed for yield $(Y)$

$$
Y_{n}=F_{n}(N)=a_{n}+b_{n} N+c_{n} N^{2} \quad(n=I, m, h)
$$
maximizes the payoff for area $n$ is

$$
N_{n}{ }^{*}=\left[1 /\left(-2 c_{n}\right)\right]\left[b_{n}-\left(P_{N} / P_{y}\right)\right] \quad(n=I, m, h) .
$$


1 The payoff from applying the optimal rate $\left(N_{n}{ }^{*}\right)$ in area $n$ is

3 The overall payoff under site-specific crop management $\left(\Pi^{s *}\right)$ is the sum of the payoffs for each 4 area,

$$
\pi_{n}{ }^{*}=P_{y} F_{n}\left(N_{n}{ }^{*}\right)-P_{N} N_{n}^{*}(n=l, m, h) .
$$

$$
\Pi^{s *}=\pi_{1}^{*} \times A_{l}+\pi_{\mathrm{m}}{ }^{*} \times A_{m}+\pi_{\mathrm{h}}{ }^{*} \times A_{h}
$$

where $A_{h}, A_{m}$ and $A_{l}$ are the areas of low, medium and high yield regions.

The alternative to site-specific management is to apply a uniform rate, $\mathrm{N}^{\cup}$, across all areas regardless of site-specific yield potential. The payoff to applying $\mathrm{N}^{\cup}$ per unit of land in area $n$ is

$$
\pi_{n}^{U}=P_{y} F_{n}\left(N^{U}\right)-P_{N} N^{U}(n=l, m, h)
$$

so the net return of uniform management across the field ( $\left.\Pi^{\cup}\right)$, is

$$
\Pi^{\cup}=\pi_{l}^{U} \times A_{l}+\pi_{m}{ }^{u} \times A_{m}+\pi_{h}^{U} \times A_{h}
$$

The benefit $(B)$ of site-specific crop management relative to uniform management is the difference between (4) and (5):

$$
B=\Pi^{s *}-\Pi^{U}=\left(\pi_{1}{ }^{*}-\pi_{l}^{U}\right) \times A_{l}+\left(\pi_{m}{ }^{*}-\pi_{m}{ }^{U}\right) \times A_{m}+\left(\pi_{h}{ }^{*}-\pi_{h}{ }^{U}\right) \times A_{h}
$$

This gives us our measure of the gross benefit of site-specific crop management relative to uniform rates. In evaluating the overall performance of site-specific management, extra costs would also have to be considered, but here we focus only on the benefit. We can express this benefit relative to the maximum net return under site-specific management:

$$
B_{r}=\left(\Pi^{s *}-\Pi^{U}\right) / \Pi^{s *}
$$

\section{Empirical Model Calculating Benefits from Site-Specific and Uniform Management}

Measuring the benefits of site-specific crop management requires a yield response function to nitrogen $(F(N))$ and prices. For this analysis, the base production function (in tonnes per hectare) for the medium-yield area is from Meyer-Aurich et al. (2010) for the application of nitrogen to wheat in Germany,

$$
Y_{m}=F_{m}(N)=2.144+0.0265 N-0.00005 N^{2}
$$


1 The prices are $200 €$ per tonne for winter wheat and $1.5 €$ per $\mathrm{kg}$ for nitrogen. Plugging this

2 information into equation (7), the optimal application rate for nitrogen on the medium-yield regions 3 is

$$
N_{m}{ }^{*}=(1 /(-2 \times(-0.00005)) \times(0.0265-1.5 / 200)=190 \mathrm{~kg} \text { per ha }
$$

5 The yield response for the low (high) management zone is assumed to involve a $20 \%$ reduction

6 (increase) in the parameters $a_{1}$ and $b_{1}\left(a_{h}\right.$ and $\left.b_{h}\right)$ compared to the base production function given in 7 (14). Thus, the response function for the low-yield are is

8

9 giving $N_{l}^{*}=137 \mathrm{~kg}$ per ha. For the high-yield areas, optimal values are plotted in Figure 4. $\mathrm{kg} / \mathrm{ha}$, the same as for the medium management region.

$$
F_{1}(N)=1.715+0.0212 N-0.00005 N^{2},
$$

$$
F_{h}(N)=2.575+0.0318 N-0.00005 N^{2},
$$

giving $N_{h}{ }^{*}=243 \mathrm{~kg}$ per ha. Plugging in these rates into equation (8) provides the value of the payoff of applying the optimal rate in each area $\left(\pi_{\mathrm{n}}{ }^{*}\right)$. These payoff functions along with their associated

Given the wide variation in optimal nitrogen rate across the three discrete parts of the field illustrated in Figure 4, it might be expected that the benefits of adjusting nitrogen rates across the three parts of the field would be high. The process for calculating the economic benefits of sitespecific management is illustrated in Figure 5. In calculating the results, we assume that the field consists of $50 \%$ medium yield, and the remaining area split evenly between the low and high management zones. Thus, the optimal $\mathrm{N}$ rate for a uniform application $\left(N^{\mathrm{*}}\right)$ in this case is 190

In the low-yielding area, the benefit of reducing the fertilizer rate from $\mathrm{N}^{\mathrm{U} *}(190)$ to $\mathrm{N}_{1}{ }^{*}$ (137) equals $\pi_{1}{ }^{*}-\pi_{1} \cup(531 €-503 €)$, the net return at $N_{1}{ }^{*}$ minus the net return at $\mathrm{N}^{\mathrm{U}}$ (see Figure 5). Because of the flatness of the payoff function, the proportional increase in net return is much less than the proportional reduction in input level. Similarly, the benefit of increasing the fertilizer rate from $N^{U *}(190)$ to $N_{l}{ }^{*}(243)$ equals $\pi_{h}{ }^{*}-\pi_{h}{ }^{U}(1105 €-1077 €)$, the net return at $N_{h}{ }^{*}$ minus the net return at $\mathrm{N}^{\mathrm{*}}$. Again, the increase in net return is small relative to the percentage increase in 
1 fertilizer rate. Finally, in the medium-yielding area, there is no gain in net return under site-specific management because, in this example, the optimal input rate for this area is the same as the optimal uniform input rate, $\mathrm{N}^{\mathrm{U}}=\mathrm{N}_{\mathrm{m}}{ }^{*}(=190)$ so $\pi_{\mathrm{m}}{ }^{*}=\pi_{\mathrm{m}}{ }^{\mathrm{U}}(=790 €)$. The gross benefit of site-specific crop management relative to uniform management (B) as given by (12) is $14 €$ per ha and this benefit relative to the maximum net return under site-specific management $\left(B_{r}\right)$ from (13) is 0.018.

The process of calculating the optimal rates for each management zone under site-specific management $\left(\mathrm{N}_{\mathrm{n}}{ }^{*}\right)$ and for the field under uniform management $\left(\mathrm{N}^{\mathrm{*}}\right)$ and the corresponding payoffs to those rates are repeated for alternative scenarios:

a) Distribution of Management Zones. The base case has a symmetrical distribution of the areas of low, medium and high yielding zones $(25,50,25 \%$, respectively), which is realistic in many cases (Rogers et al. 2016). However, the empirical measurements presented by Rogers et al. (2016) also include different distributions. For this reason, we also simulate results for a uniform distribution $(33,33,33 \%)$ and a skewed distribution $(25,25,50 \%)$. It is expected that giving more weight to the high and/or low yielding zones, will increase the benefits from adjusting input rates away from the uniform rate.

b) Yield Variance- The base case assumes the change in the $a$ and $b$ parameters of the quadratic response function are $20 \%$ from the production function given by equation (14). An increase in variance is imposed by increasing the change in these parameters to $30 \%$ and $40 \%$.

c) Flatness of the Payoff Curves. The payoff curves in Figures 4 and 5 are not particularly flat compared to some examples (e.g. Pannell 2006). Halving the $c$ parameter of the response function from -0.00005 to -0.000025 increases the flatness. The $a$ parameter is increased to give the same yield in medium-yield zones at a nitrogen rate of $200 \mathrm{~kg} / \mathrm{ha}$. The range of yields is the same as for the base case. 


\section{Assessing the Performance of Flatness Metrics}

2 As noted earlier, the benefits of variable-rate site-specific management decrease with the flatness of

3 the payoff function. Consequently, an appropriate flatness metric might be useful for to indicating

4 the value of changing the nitrogen application rate for each management zone as compared to

5 applying a single rate over the whole field.

6

7

An aggregate payoff curve is calculated for each of the scenarios listed above. The aggregate payoff is the combination of the low, medium and high payoff curves weighted by area. Continuing with the example used above with $25 \%$ share in both low and high management zones, than the aggregate payoff curve is the payoff for the medium-yielding region. This aggregate payoff is used to illustrate how the alternative measures of flatness are calculated.

IR95 involves first taking $95 \%$ of the maximum payoff of $790 €$, which is $750 €$. This payoff is associated with a nitrogen rate of $127 \mathrm{~kg} / \mathrm{ha}\left(\mathrm{N}_{\underline{d}}\right)$ and a $\mathrm{N}$ rate $252 \mathrm{~kg} / \mathrm{ha}\left(\mathrm{N}_{\underline{u}}\right)$ while the rate that maximizes return was $190 \mathrm{~kg} / \mathrm{ha}\left(\mathrm{N}^{*}\right)$. Thus, the

$R C$ requires first choosing a high application rate (i.e. $=400$ ) and multiplying this by the maximum value of the aggregate payoff $(790 €)$ to get the area under the rectangle illustrated in Figure $2(316 €)$. The area under the aggregate payoff curve is estimated as the sum of the aggregate payoff curve for each level of nitrogen use up to arbitrary high level of $400 \mathrm{~kg} / \mathrm{ha}$, which in the example above is 263 . Thus, the $R C$ is

\section{SDS requires estimating the slope of the payoff function associated with each of the} management zones at the optimal uniform input rate $\left(\mathrm{N}^{\mathrm{U}}\right)$, which is $190 \mathrm{~kg} / \mathrm{ha}$ in the base case scenario. The payoff function for each management zone evaluated at $\mathrm{N}^{\mathrm{*}}$ is

$$
\pi_{\mathrm{n}}{ }^{\mathrm{*}}=P_{\mathrm{y}} F_{n}\left(N^{U^{*}}\right)-P_{N} N^{U *}(n=\mathrm{I}, \mathrm{m}, \mathrm{h}) .
$$

The slope is thus

$$
\pi_{n}^{\prime}{ }^{U *}=P_{y}\left(F_{n}\left(N^{U *}\right) / N^{U *}\right)-P_{N}=P_{y}\left(b_{n}+2 c_{n} N^{U *}\right)-P_{N} \quad(n=I, m, h),
$$


1 which in the base case scenario results in the following slopes for each management zone:

$$
\pi_{1}^{\prime}{ }^{* *}=-1.06, \pi_{m}^{\prime}{ }^{u *}=0, \text { and } \pi_{h}^{\prime}{ }^{u *}=1.06
$$

3 The standard deviation of these slopes across the field, SDS, is

To test the overall suitability of the three flatness metrics as indicators of the benefits of site-specific crop management, we estimate the correlations between each metric and the actual benefits. We also correlate the metrics with each other. The correlations were calculated over 18 simulations (3 yield zone distributions $\mathrm{x} 3$ yield variances $\mathrm{x} 2$ flatness measures).

\section{Influence of Externality Costs on Benefits and Flatness Metrics}

Finally, we examine the influence of an environmental externality on the benefits of site-specific management, and the performance of the three metrics in capturing this influence. Rogers et al. (2016) showed that $R C$ increases when external environmental costs are internalised (e.g. a pollution tax is levied on farmers for each unit of a nutrient that leaves their property). They interpreted this as meaning that site-specific crop management is more beneficial when the environmental impacts of nutrient use are accounted for.

Two relationships between nitrogen application rate and external cost are tested:

a) The external cost (EC) is set at $33 \%$ of the purchase and application cost of nitrogen. It is assumed that the external cost is the same for each unit of nitrogen and the same for each yield zone.

b) In the second scenario, it is assumed that external cost increases quadratically, with the function calibrated to give the same external cost as the linear function at a nitrogen rate of $200 \mathrm{~kg} / \mathrm{ha}$. 


\section{Results}

2 The results for the base case scenario illustrated above with a symmetrical distribution of three yield

3 zones within the field are listed in the first column results of Table 1 . The potential gain in profit

4 from switching from a uniform fertilizer rate to a site-specific one is $€ 14$, or $1.8 \%$. The flatness of the

5 three payoff functions means that the changes in profit are much smaller percentages that the

6 changes in fertilizer rates, as shown in Figures 4 and 5 . The IR95 indicator is 0.66 , meaning that the

7 range of input rates that give profits at least $95 \%$ of the optimal uniform rate is $66 \%$ of the optimal

8 uniform rate. $R C$ is 0.17 and $S D S$ is 0.75 - values that that are not helpful in themselves but may be

9 useful when compared across scenarios.

The benefits of precision depend on the variability of yields across different zones of the

field. The second and third sets of results are for the scenarios with high and very high yield variance under the symmetric management zones (Table 1). The benefits of precision ( $B$ or $\left.B_{r}\right)$ increase with yield variance; higher yield variance means that optimal site-specific input rates are more variable and the slopes of the payoff functions at the optimal uniform rate are higher, meaning that input rate adjustments make a bigger difference to payoffs. Although the economic gains from precision are more than three times larger under the very-high yield-variance scenario compared with the base case, they are still relatively modest at $7.1 \%$, reflecting the strong influence of payoff-function flatness.

Of the three metrics of payoff-function flatness, only SDS reflects the increasing benefits of site-specific inputs under increasing yield variance (Table 1). Both $R C$ and $I R 95$ are unchanged across the three yield-variance scenarios because both are calculated from the mean payoff function, which is unchanged across these scenarios. SDS is positively correlated, although not perfectly, with $B$ and $B_{r}$ (Table 2).

The benefits of site-specific crop management also depend on the flatness of the payoff curves. The payoff curves in Figures 4 and 5 are not particularly flat compared to some examples 
1 (e.g. Pannell 2006). Increasing the flatness of the payoff function reduces the benefits of site-specific

2 management at each level of yield variability by about $11 \%$ (rows 4-6 in Table 1 ).

3 All three of the indicators overstate the impact of flatness on the benefits of precision. IR95 and SDS

4 both change by $33 \%$ in response to the increased flatness, while $R C$ falls by $51 \%$, compared with the

5 actual change in benefits of $11 \%$.

6

7

Altering the area distribution of the management zones alters the benefits of site-specific management and the effectiveness of the flatness metrics. Giving more weight to the high- and/or low-yielding zones increases the benefits from adjusting input rates away from the uniform rate. For the uniform distribution, the benefits rise by $33 \%$, while for the skewed result they rise by almost 40\%. $\left(B_{r}\right.$ rises by a smaller proportion because the skew towards high-yielding zones means that expected profit is higher, so the gain relative to expected profit is lower).

For the uniform distribution, SDS understates increase in benefits from precision relative to the symmetrical distribution. However, $R C$ and $I R 95$ fail to detect any benefit at all. For the skewed distribution, the change in SDS relative to the base case is about half of $B$, while IR95 detects almost no benefit, and $R C$ incorrectly indicates a reduction in benefits from precision.

Table 2 gives the correlation between each of the three flatness metrics and the benefits of site-specific crop management across the 18 scenarios presented in Table 1. As expected, there is a high degree of correlation between the absolute and relative benefits of precision. However, the two current measures of flatness (IR95 and RC) are poorly correlated with the two benefit values. In contrast, the benefits of site-specific management are highly correlated with the SDS indicator, proposed in this study. IR95 and $R C$ are highly correlated with each other and both are moderately correlated with SDS.

The effects of the external costs on payoffs for each management zone are shown in Figure 6, using quadratically increasing external costs. The solid lines represent the new payoff functions once external costs are subtracted from the farmer's private payoff functions. Thus the solid lines 
1 indicate the optimal nitrogen rates from the perspective of society as a whole, rather than for the

2 farmer.

3

4

\section{Discussion} costs.

The impact of including the external cost from nitrogen application on the benefits of sitespecific management and the performance of the three flatness metrics are listed in Table 3. For linear externality costs, there is no change in the benefits of variable-rate site-specific management relative to the base case in Table 1, irrespective of the yield variance. This is correctly reflected in the results for SDS, which are unchanged from Table 1, but IR95 and especially $R C$ incorrectly indicate that the benefit from site-specific management has increased as a result of accounting for external

For quadratic external costs, there are modest decreases in the benefits of variable-rate sitespecific management relative to the base case. This is undetected by SDS, which has the same results as for the base case. Rather than showing a decrease, $I R 95$ and $R C$ incorrectly suggest that there are increases in the benefits of site-specific management as a result of internalising quadratic external costs. The increase for $R C$ (from 0.17 to 0.27 ) is especially large in relative terms and highly misleading if $R C$ is used to identify cases where site-specific management is most beneficial.

The benefits from site-specific management for the application of nitrogen to wheat are small; the relative increase in net returns range from $2 \%$ in the base case to approximately $9 \%$ in the extreme case with very high yield variance and an even distribution of land area across management zone types, although we consider the latter scenario to be unrealistic in practice. The results are consistent with the lack of adoption of precision agriculture technologies (OECD 2016), particularly compared to the very high adoption rate of technologies using other smart farming innovations such as auto-steer (Erickson et al. 2017). Increasing the degree of heterogeneity in the field, increases the benefits of site-specific management but the results suggests that the enhanced returns from 
1 precision are unlikely to cover the costs of the technology under many situations unless capital costs decrease significantly.

A major reason for the relatively small benefits from site-specific application of nitrogen is the flatness of the payoff curve showing the relationship between nitrogen use and the net returns from varying the rate by management zone. Note the use of the quadratic response function, which allows yield to fall if excessive nitrogen is applied, will result in more curvature of the payoff function than other commonly used yield response functions, such as a linear plateau or Mitscherlich-Baule. The motivation for the research was to assess whether measuring the degree of flatness of payoff curves could identify the situations where the benefits of site-specific crop management are most likely to be high. The previously used measures of flatness, IR95 and $R C$, are highly unsuitable to use as indicators of the benefits of variable-rate site-specific crop management. IR95 was not originally proposed as an indicator for decision-making. It may still be useful is in conveying the concept of the payoff curve being flat by highlighting the range of inputs for which net returns are only $5 \%$ less than the optimal.

Both IR95 and RC fail to capture frequency distribution of the payoff curves within the field, which is an important determinant of the benefits of variable-rate technologies. In addition, $R C$ performs poorly in part because the range of input rates it uses to measure curvature is not the right range. For low yielding areas, only curvature on the up-side matters, and only up to the optimal uniform input rate. For high-yielding areas, only curvature on the down-side matters, and only down to the optimal uniform input rate. For average yielding areas (or at least for areas that have optimal input rates close to the optimal uniform rate), curvature doesn't matter at all. Even if we limited the range used to calculate $\mathrm{RC}$ to these ranges, it still would not make sense because what actually matters is the loss of payoff at the optimal uniform rate. What is happening at rates between the optimal rate for low-yielding areas and the optimal uniform rate is irrelevant, because those inbetween rates are not actually applied. 
the higher the slope of the payoff function in each management zone, the greater the benefits from site-specific management. On the other hand, data requirements to apply the SDS indicator are high. As a minimum, it needs information about yields at a near-optimal input rate and another moderately different rate, for various areas in the field. If an analyst had sufficient information to calculate the SDS, it would be only a small step to calculate the economic benefits of variable-rate technology using the economic model presented earlier. Like IR95, its main contribution in practice maybe to influence perceptions and understanding.

Having explored these three indicators of the flatness of payoff functions, our inclination is to focus on exploring efficient ways of applying the economic model that estimates the financial benefits of variable rate technologies, rather than relying on an indicator. In the near future innovative smart farming technologies might enable farmers to conduct on farm trials to test sitespecific crop response to inputs at low cost.

\section{References}

Anderson, J. R. (1975). Programming For Efficient Planning Against Non-Normal Risk. Australian Journal of Agricultural and Resource Economics, 19(2), 94-107.

Bachmaier, M., Gandorfer, M. (2009). A conceptual framework for judging the precision agriculture hypothesis with regard to site-specific nitrogen application. Precision Agriculture 2008, 10, 95.

Erickson, B., Lowenberg-DeBoer, J., \& Bardford, J. (2017). 2017 Precision agriculture dealership survey. Dept of Agriculture Economics and Agronomy, Purdue University, December.

Lawes, R. A., \& Robertson, M. J. (2011). Whole farm implications on the application of variable rate technology to every cropped field. Field Crops Research, 124(2), 142-148.

Meyer-Aurich, A., Weersink, A., Gandorfer, M., \& Wagner, P. (2010). Optimal site-specific fertilization and harvesting strategies with respect to crop yield and quality response to nitrogen. Agricultural Systems, 103(7), 478-485.

Organisation for Economic Co-operation and Development (OECD), (2016). Farm Management Practices to Foster Green Growth, OECD Green Growth Studies, OECD Publishing, Paris. Available at: http://dx.doi.org/10.1787/9789264238657-en

Pannell, D. J. (2006). Flat earth economics: the far-reaching consequences of flat payoff functions in economic decision making. Review of Agricultural Economics, 28(4), 553-566. 
1 Rogers, A., Ancev, T., \& Whelan, B. (2016). Flat earth economics and site-specific crop management: 2 how flat is flat?. Precision agriculture, 17(1), 108-120.

3 Weersink, A., Fraser, E., Pannell, D., Duncan, E., \& Rotz, S. (2018). Opportunities and challenges for 4 Big Data in agricultural and environmental analysis. Annual Review of Resource Economics, 10, 19-37

\section{Acknowledgements}

7 Pannell acknowledges the financial support provided by the ARC Centre of Excellence for

8 Environmental Decisions. Gandorfer acknowledges support from the Bavarian State Ministry of 9 Food, Agriculture, Forestry. Financial support from the Canada First Research Excellence Fund and 10 the Ontario Ministry of Agriculture, Food, And Rural Affairs (30242) is gratefully acknowledged by 11 Weersink. 
Table 1. The economic benefit of site specific management and the performance of the three metrics measuring flatness for various scenarios

\begin{tabular}{|c|c|c|c|c|c|c|c|}
\hline \multicolumn{3}{|c|}{ Scenario } & \multicolumn{2}{|c|}{ Benefit of Site Specific Management } & \multicolumn{3}{|c|}{ Flatness of Payoff Function Metrics } \\
\hline $\begin{array}{l}\text { Distribution of } \\
\text { low-med-high }\end{array}$ & Yield Variance & $\begin{array}{c}\text { Flatness of } \\
\text { Payoff }\end{array}$ & Absolute $B(€ / \mathrm{ha})$ & Relative $B_{r}$ & IR95 & $R C$ & SDS \\
\hline \multirow[t]{6}{*}{$25-50-25$} & Base & Base & 14.0 & 0.018 & 0.66 & 0.17 & 0.75 \\
\hline & High & Base & 31.6 & 0.040 & 0.66 & 0.17 & 1.12 \\
\hline & Very high & Base & 56.2 & 0.071 & 0.66 & 0.17 & 1.50 \\
\hline & & & & & 000 & & 0 \\
\hline & $\begin{array}{l}\text { Base } \\
\text { High }\end{array}$ & $\begin{array}{l}\text { Flatter } \\
\text { Flatter }\end{array}$ & $\begin{array}{l}12.5 \\
28.1\end{array}$ & $\begin{array}{l}0.016 \\
0.036\end{array}$ & $\begin{array}{l}0.00 \\
0.88\end{array}$ & 0.00 & 0.75 \\
\hline & Very high & Flatter & 49.9 & 0.063 & 0.88 & 0.08 & 1.00 \\
\hline \multirow[t]{7}{*}{ 33-33-33 } & Base & Base & 18.7 & 0.024 & 0.66 & 0.17 & 0.87 \\
\hline & High & Base & 42.1 & 0.053 & 0.66 & 0.17 & 1.30 \\
\hline & Very high & Base & 74.9 & 0.095 & 0.66 & 0.17 & 1.74 \\
\hline & & & & & & & \\
\hline & Base & Flatter & 16.6 & 0.021 & 0.88 & 0.08 & 0.58 \\
\hline & High & Flatter & 37.5 & 0.047 & 0.88 & 0.08 & 0.87 \\
\hline & Very high & Flatter & 66.6 & 0.084 & 0.88 & 0.08 & 1.16 \\
\hline \multirow[t]{6}{*}{$25-25-50$} & Base & Base & 19.3 & 0.022 & 0.65 & 0.15 & 0.88 \\
\hline & High & Base & 43.5 & 0.048 & 0.64 & 0.15 & 1.32 \\
\hline & Very high & Base & 77.3 & 0.082 & 0.64 & 0.14 & 1.76 \\
\hline & Base & Flatter & 17.2 & 0.020 & 0.84 & 0.08 & 0.59 \\
\hline & High & Flatter & 38.6 & 0.043 & 0.82 & 0.08 & 0.88 \\
\hline & Very high & Flatter & 68.7 & 0.074 & 0.80 & 0.08 & 1.17 \\
\hline
\end{tabular}


Table 2. Correlation matrix for benefit of precision $(B)$, relative benefit of precision $\left(B_{r}\right)$, Input Range 95 (IR95), Relative Curvature $(R C)$ and standard deviation of the slopes of site-specific payofffunctions at the optimal uniform input rate (SDS).

\begin{tabular}{|l|c|r|r|r|r|}
\hline & \multicolumn{2}{|c|}{ Benefits of Precision } & \multicolumn{3}{|c|}{ Flatness Metric } \\
\hline & $B$ & \multicolumn{1}{|c|}{$B_{r}$} & \multicolumn{1}{|c|}{ IR95 } & \multicolumn{1}{c|}{ SDS } \\
\hline$B$ & 1 & 1 & & & \\
\hline$B_{r}$ & 0.99 & -0.13 & 1 & & \\
\hline$I R 95$ & -0.16 & 0.09 & -0.95 & 1 & \\
\hline$R C$ & 0.07 & 0.86 & -0.60 & 0.53 & 1 \\
\hline$S D S$ & 0.87 & & & & \\
\end{tabular}


Table 3. The economic benefit of precision and the performance of the three metrics measuring flatness in the presence of external costs due to nitrogen application. The distribution of low-, medium- and high-yield areas is assumed to be 25-50-25.

\begin{tabular}{|c|c|c|c|c|c|c|}
\hline \multicolumn{2}{|c|}{ Scenario } & \multicolumn{2}{|c|}{ Benefits of Precision } & \multicolumn{3}{|c|}{ Flatness Metric } \\
\hline $\begin{array}{l}\text { Externality } \\
\text { Costs }\end{array}$ & $\begin{array}{l}\text { Yield } \\
\text { Variance }\end{array}$ & $B(€ /$ ha $)$ & $B_{r}$ & IR95 & $R C$ & SDS \\
\hline Linear & Base & 14.0 & 0.020 & 0.72 & 0.21 & 0.75 \\
\hline & High & 31.6 & 0.045 & 0.72 & 0.21 & 1.12 \\
\hline & Very High & 56.2 & 0.080 & 0.72 & 0.21 & 1.50 \\
\hline Quadratic & Base & 11.2 & 0.016 & 0.70 & 0.27 & 0.75 \\
\hline & High & 25.3 & 0.035 & 0.70 & 0.27 & 1.12 \\
\hline & Very High & 44.9 & 0.063 & 0.70 & 0.27 & 1.50 \\
\hline
\end{tabular}




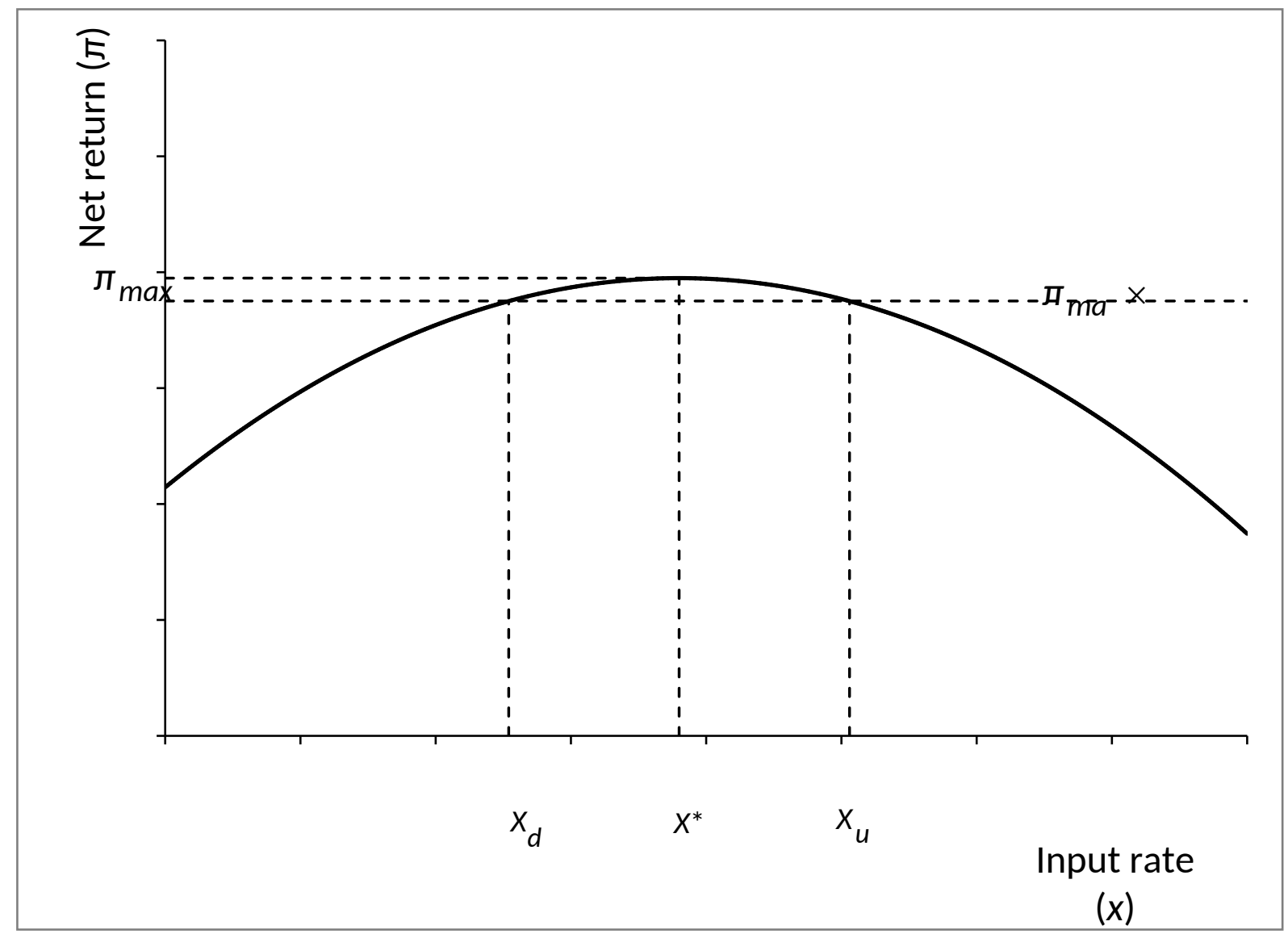

Figure 1. An example payoff function showing the range of input levels giving a payoff at least $95 \%$ of the maximum payoff, $\pi_{\max }$. 


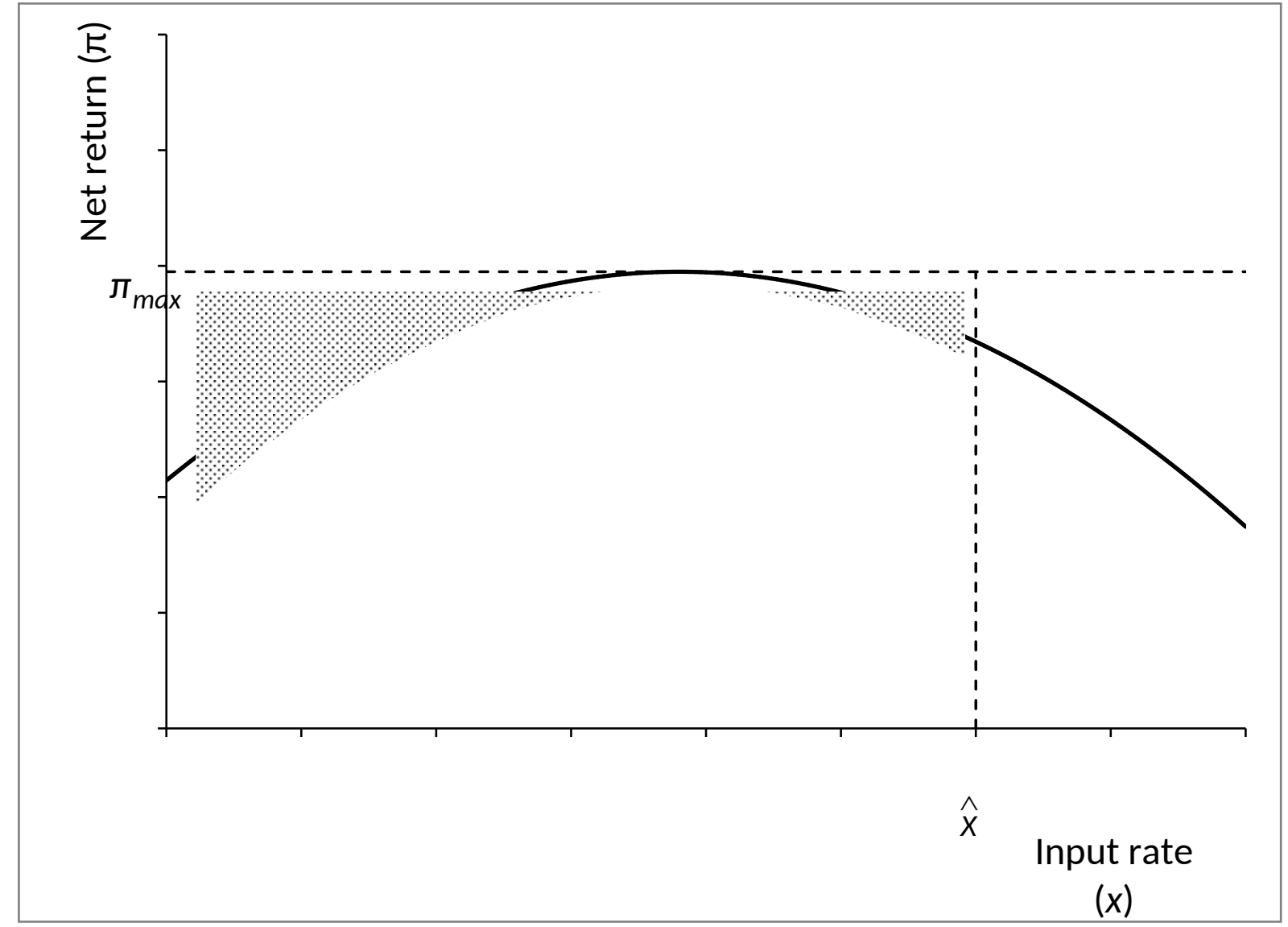

Figure 2. The Relative Curvature of the payoff function is defined as the shaded area divided by the area of the rectangle. 


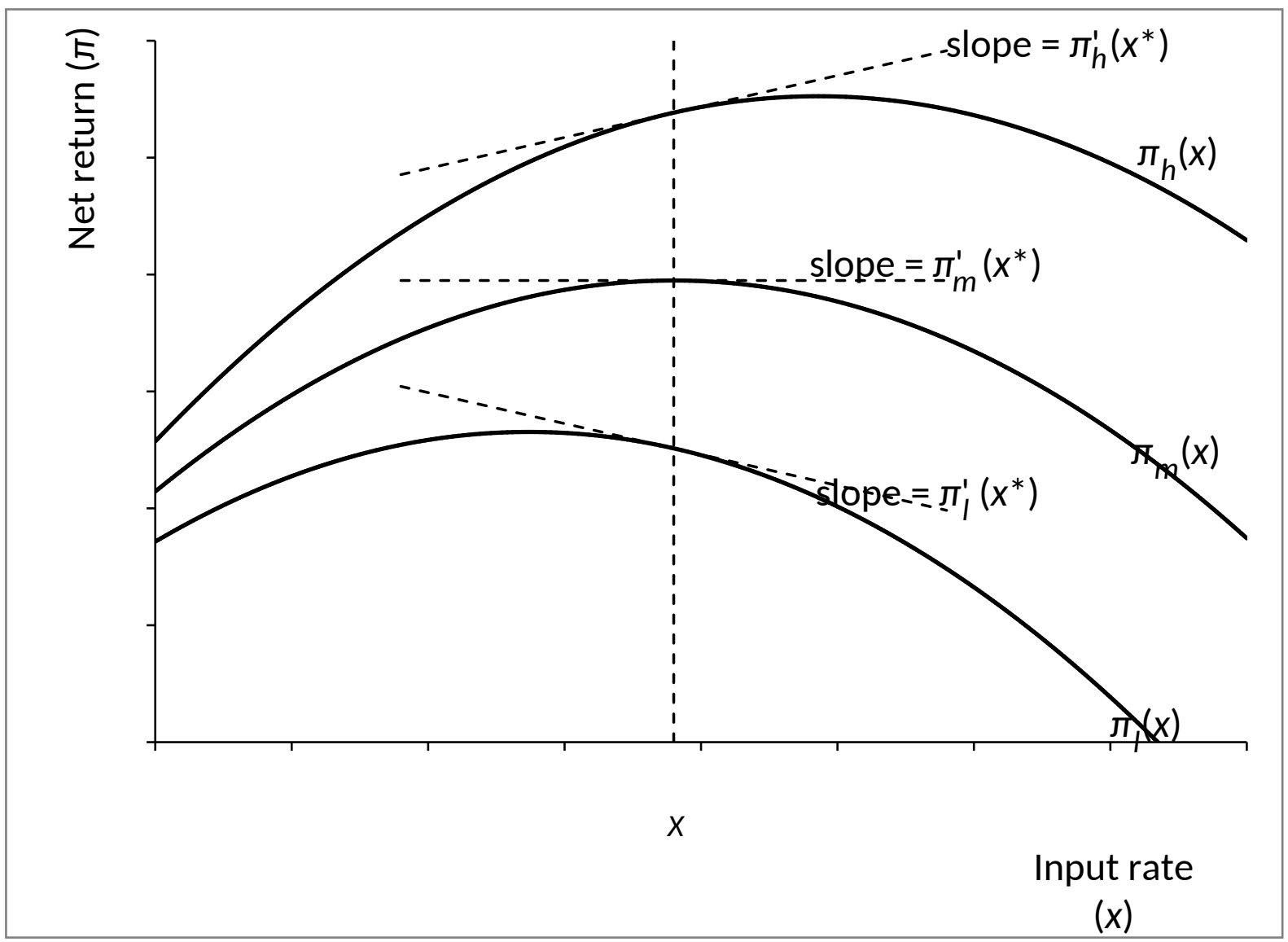

Figure 3. Illustration of the slopes used to calculate SDS for a case where there are three discrete regions in a field with payoff functions $\pi_{l}(x), \pi_{m}(x)$ and $\pi_{h}(x)$. 


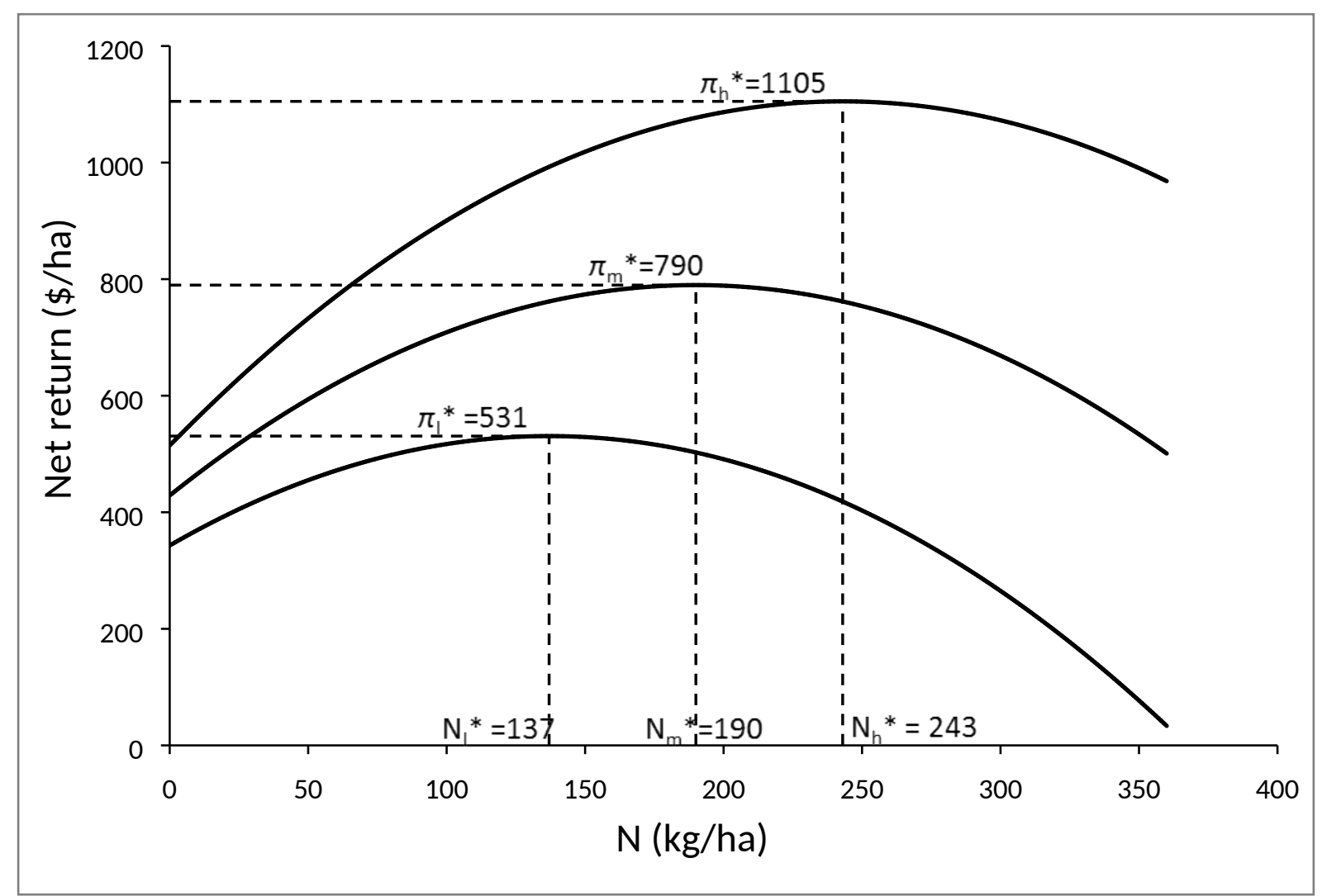

Figure 4. Optimal nitrogen fertilizer rates, $N_{l},{ }^{*}, N_{m}{ }^{*}$, and $N_{h}{ }^{*}$, for three payoff functions occurring within parts of a field. 


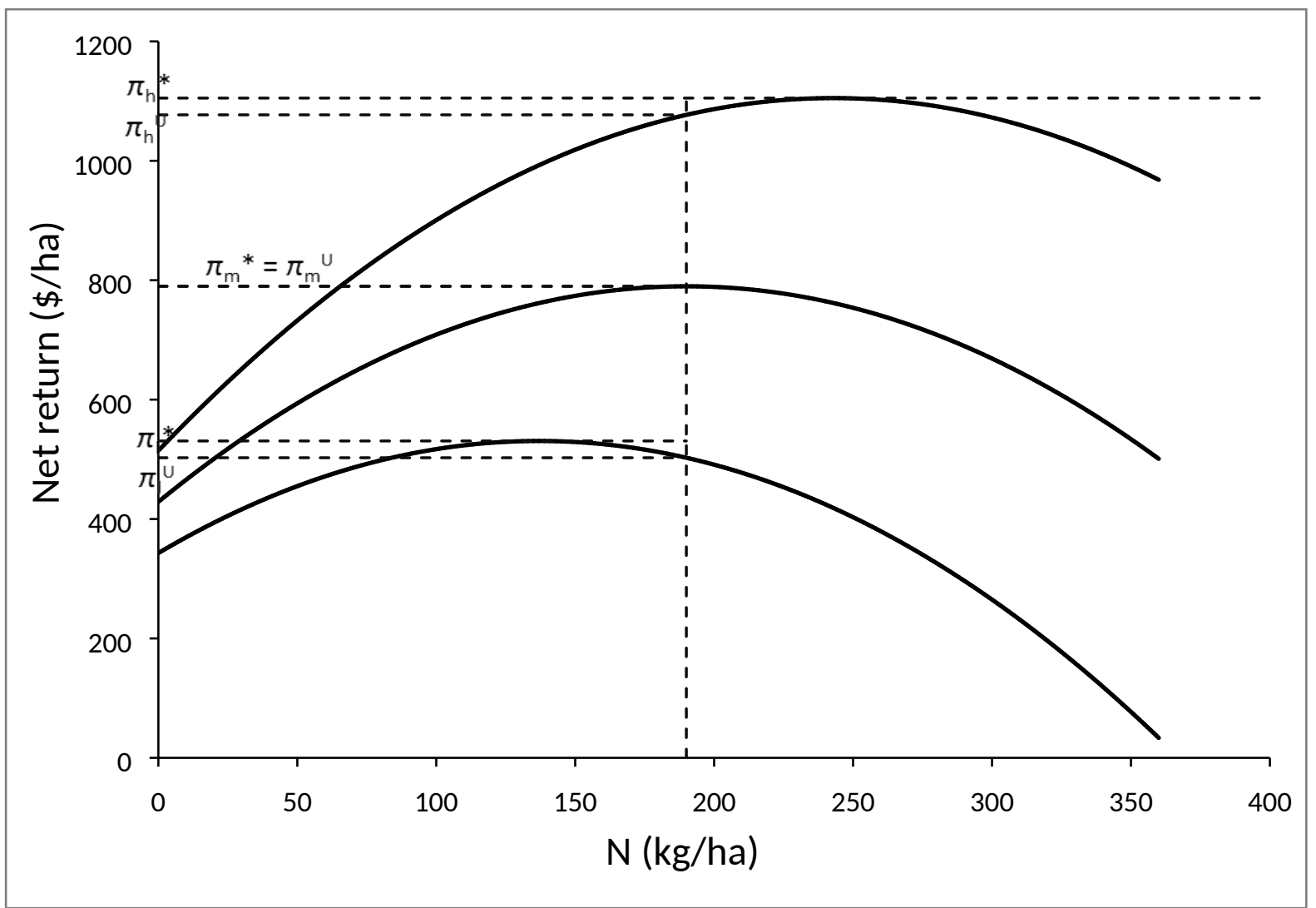

Figure 5. The benefits of site-specific crop management within three areas of a field with different payoff functions. 


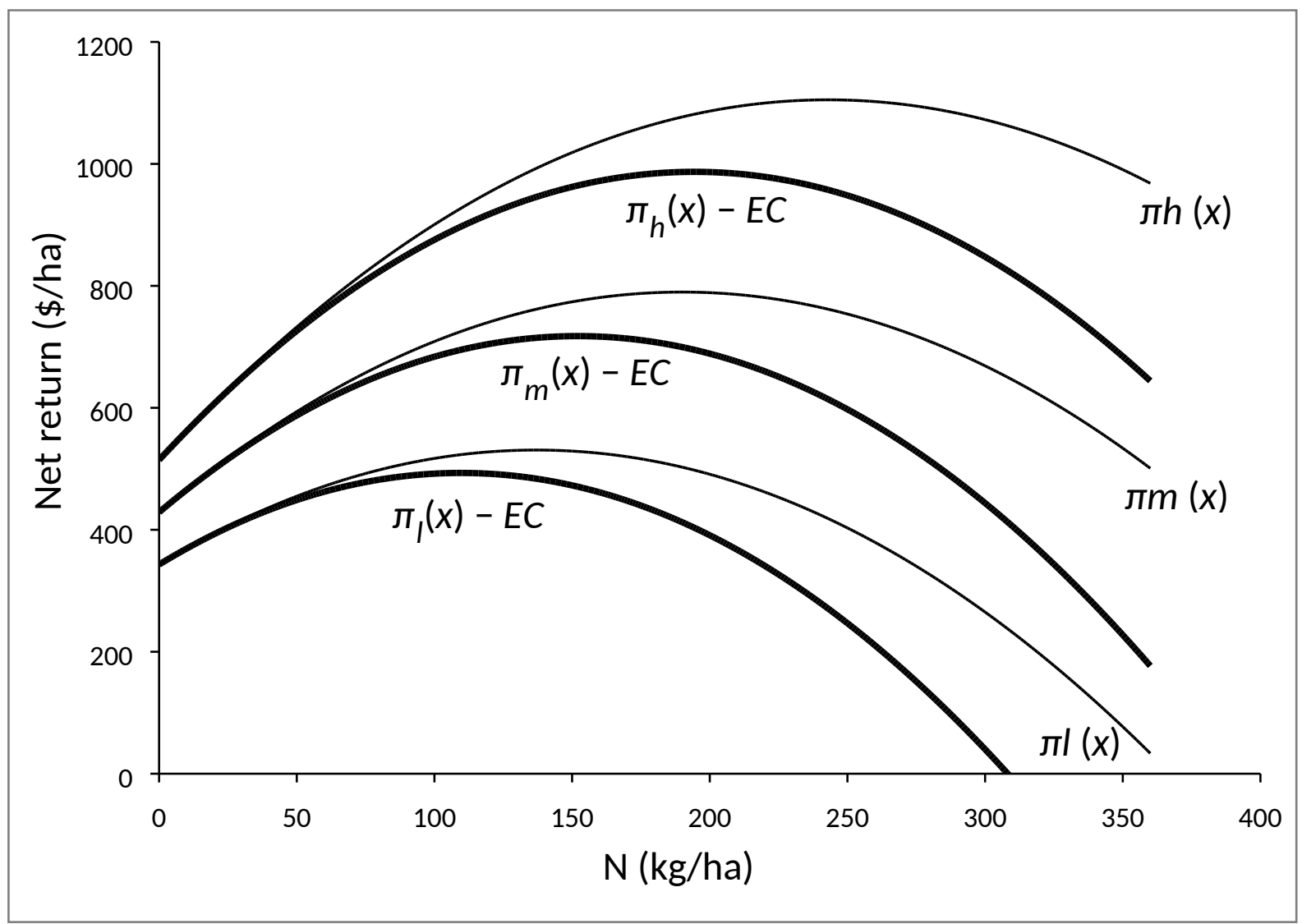

Figure 6. Effect of external costs of nutrient pollution on the payoff functions for each yield zone, assuming that external costs increase quadratically with nitrogen rate and are the same for each yield zone. 